\title{
Evaluación de la colmatación de una membrana mediante medidas eléctricas y de la composición química de su superficie
}

\author{
J.BENAVENTE, M.J.ARIZA Y A.CAÑAS. \\ Grupo Fenómenos Electrocinéticos: Caracterización Electrica y de Transporte en Membranas e Interfases. \\ Dpto. Física Aplicada, Facultad de Ciencias, Universidad de Málaga. 29071 Málaga.
}

\begin{abstract}
La adsorción de macromoléculas cargadas sobre la superficie de una membrana en procesos de microfiltración produce la colmatación (o ensuciamiento) de la membrana y, por tanto, una disminución del flujo a través de la membrana. La colmatación puede ser facilmente detectada por la variación de: i) parámetros electroquímicos como la resistencia eléctrica y la permeabilidad salina, directamente relacionados con la porosidad de las membranas; ii) las concentraciones atómicas de los elementos característicos de la superficie de la membrana, determinadas mediante espectroscopía de fotoelectrones de rayos $\mathrm{X}$ (XPS). Para membranas comerciales de alúmina (Anopore ${ }^{\mathrm{TM}}, 0.1 \mu \mathrm{m}$ de tamaño de poro) en contacto con disoluciones que contienen $0.5 \mathrm{~g} / 1$ de seroalbúmina de bobino (BSA), los parámetros eléctroquímicos indican una disminución en la porosidad de la membrana del 50\%, aproximadamente, despues de estar 72 h en contacto con una disolución con proteína. Los resultados de XPS indican: i) que la capa de proteína adsorbida sobre la superficie de la membrana no aumenta linealmente con el tiempo de interacción membrana-proteína, sino que se produce fundamentalmente durante las primeras 24 h.; ii) la no uniformidad de la capa de proteína adsorbida sobre la membrana de alúmina.
\end{abstract}

Palabras clave: resistencia eléctrica y difusión en membranas de alúmina, adsorción de proteínas, espectroscopía de fotoelectrones de rayos X (XPS).

Evaluation of membrane fouling by electical and surface chemical composition measurements.

Adsorption of charged macromolecules on the surface of a membrane in microfiltration processes produces membrane fouling and, as a result of this, a reduction in the flow across the membrane. Membrane fouling can be easyly detected by variation of: i) electrochemical parameters such as electrical resistance and salt permeability, which are directly related to the porosity of the membrane; ii) atomic concentrations of characteristic elements of the membrane surface, which can be determined by X-ray Photoelectron spectroscopy (XPS). For alumina commercial membranes (Anopore ${ }^{\mathrm{TM}}, 0.1 \mu \mathrm{m}$ pore size) in contact with salt solutions containing $0.5 \mathrm{~g} / \mathrm{l}$ bovine serum albumin (BSA), electrochemical parameters indicate a decrease around $50 \%$ for membrane porosity. XPS results show: i) the adsorbed layer of protein on the membrane surface does not directly increase depending on the membrane-protein interaction time, since most of it is adsorbed in the first 24 h.; ii) the protein layer does not uniformely cover the alumina membrane surface.

key words: electrical resistance and salt diffusion across an alumina membrane, protein adsorption, X-ray Photoelectron Spectroscopy (XPS).

\section{INTRODUCCION}

Los Procesos de Separación por Membranas, en los que la membrana actúa como una barrera selectiva que permite el paso de ciertos componentes mientras que retiene otros en la mezcla, son una alternativa clara a las técnicas convencionales de separación (1). En la mayoría de las aplicaciones industriales de los procesos de membrana tambien es necesario un flujo de disolución adecuado, por lo que la relación selectividadflujo para una membrana es un factor fundamental en su posible aplicación a un determinado proceso.

Uno de los problemas más comunes en la microfiltración/ ultrafiltración de proteínas o macromoléculas cargadas a través de membranas es la adsorción/deposición de partículas sobre su superficie (colmatación o ensuciamiento de la membrana). La colmatación de una membrana da lugar a una disminución de su porosidad efectiva y, por tanto, a una disminución del flujo de disolución que atraviesa la membrana. La colmatación depende, entre otros factores, del material membranario y del tiempo de trabajo de la membrana, pudiendo afectar significativamente a su carga efectiva y, consecuentemente, a las interacciones eléctricas membrana-disolución, lo que originaría variaciones en los parámetros electrocinéticos (2-3).
En este trabajo se estudia el efecto de la adsorción de una proteina (seroalbúmina de bovino o BSA) sobre una membrana de alúmina para microfiltración mediante la determinación de: i) parámetros electroquímicos, tales como la resistencia y la permeabilidad salina de la membrana; ii) la composición química de la superficie de la membrana, determinada mediante espectroscopía de fotoelectrones de rayos-X (XPS). La variación con el tiempo de las composiciones atómicas de ciertos elementos debido a la interacción membranaproteina, permite utilizar los resultados de XPS para monotorizar el grado de recubrimiento de la membrana por la proteina.

\section{PROCEDIMIENTO EXPERIMENTAL}

\subsection{Material}

Se ha utilizado una membrana de alúmina de microfiltración $\left(\gamma-\mathrm{Al}_{2} \mathrm{O}_{3}\right.$, Anopore $\left.{ }^{\mathrm{TM}}\right)$ de $0.1 \mu \mathrm{m}$ de tamaño de poro y 60 $\mu \mathrm{m}$ de espesor, a la que se denominará AnL; la membrana se ensució manteniéndola en contacto, durante tres días, con una disolución que contenía $0.5 \mathrm{~g} / 1$ de BSA, denominando AnF a la membrana colmatada (ensuciada). Las medidas de los pará- 


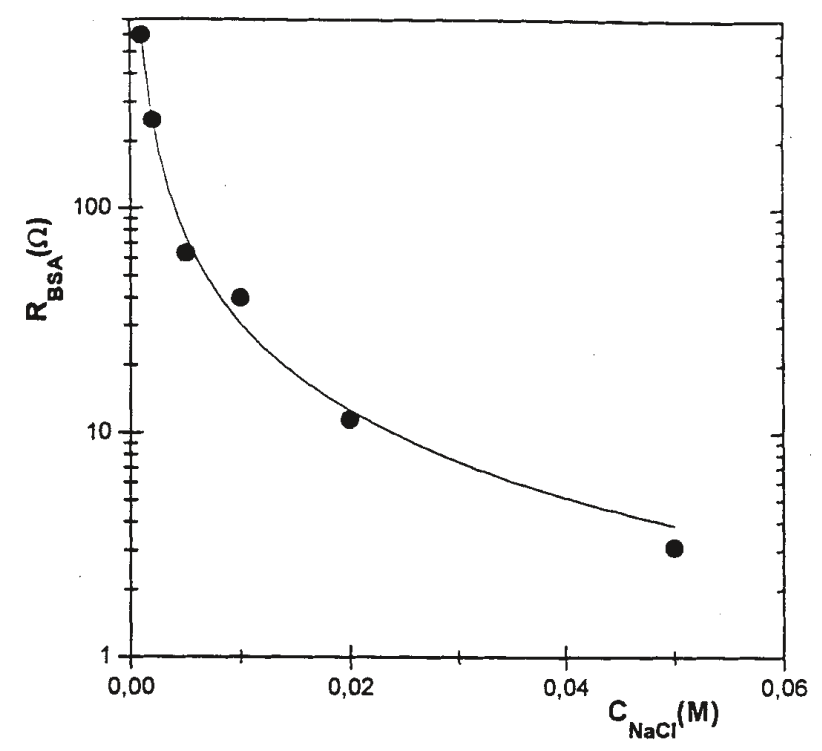

Figura 1: Variación con la concentración de $\mathrm{NaCl}$ de la resistencia en la capa de proteína adsorbida sobre la membrana.

metros electroquímicos se realizaron con disoluciones de $\mathrm{NaCl}$ a $(25.0 \pm 0.3)^{\circ} \mathrm{C}$ y $\mathrm{pH}=(6.0 \pm 0.3)$.

\subsection{Métodos experimentales}

La célula utilizada para la determinación de la resistencia eléctrica y la permeabilidad salina a través de las membranas es similar a la indicada en la literatura (4) y, basicamente, consta de dos semi-células de igual volumen entre las que se coloca la membrana, soportada entre dos juntas de silicona. El efecto de polarización de la concentración en las capas adyacentes a la membrana se minimiza agitando las disoluciones mediante un imán teflonado que gira a 500 rpm.

\subsubsection{MEDIDA DE PARÁMETROS ELECTROQUÍMICOS}

Las medidas de la resistencia eléctrica de las membranas se realizaron con un puente de alterna (Wayne Kerr, B905) a la frecuencia de $1 \mathrm{kHz}$ (5), usando electrodos de $\mathrm{Ag} / \mathrm{AgCl}$. Los valores de la resistencia se midieron a distintas concentraciones de $\mathrm{NaCl}$ (las mismas a ambos lados de la membrana), comprendidas entre $10^{-3} \mathrm{M}$ y $5 \times 10^{-2} \mathrm{M}$. La resistencia de la capa de BSA se determinó a partir de los valores de la membrana colmatada y límpia: $R_{B S A}=R_{A n F}-R_{A n L}$; los valores de las resistencias de contacto son los mismos en ambos casos, y por tanto se cancelan (5).

En las medidas de difusión, la membrana separa dos disoluciones de concentraciones diferentes: una disolución concentrada en la semi-célula $1\left(c_{1}=0.05 \mathrm{M}\right)$ e, inicialmente, una disolución diluida en la semi-célula 2 (agua destilada, $\mathrm{c}_{2}=0$ ). La evolución temporal de la concentración de la disolución en la semi-célula 2 se ha determinado mediante la variación de la conductividad (conductivímetro digital, Radiometer CDM 83).

\subsubsection{CARACTERIZACIÓN QUÍMICA DE LA SUPERFICIE}

Los espectros de fotoelectrones de rayos X (XPS) de alta resolución se han registrado en un equipo Perkin Elmer PHI 5700, usando como fuente de excitación Rayos X MgK $(15 \mathrm{kV}$, $300 \mathrm{~W})$. El analizador de electrones trabajó en el modo de ener-

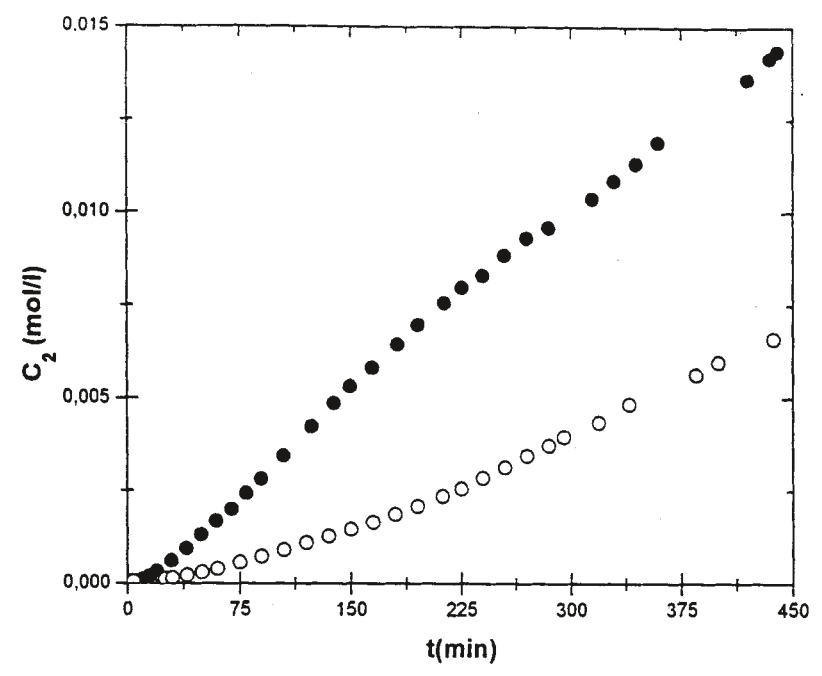

Figura 2: Variación con el tiempo de la concentración de $\mathrm{NaCl}$ en la semi-cára $2\left(\mathrm{C}_{2}\right)$, siendo $\mathrm{C}_{1}=0.05 \mathrm{M}$. (o) membrana AnL; (o) membranan AnF.

gía de paso constante a $29.35 \mathrm{eV}$ y formando un ángulo de $45^{\circ}$ con respecto a la normal de la muestra, usándose una apertura que permite registrar información de una superficie sobre la muestra de $720 \mu \mathrm{m}$ de diámetro. Con estas condiciones la señal $\mathrm{Au}_{4} \mathrm{f}_{7 / 2}$ aparece a $84.00 \mathrm{eV}$ y presenta una anchura a media altura (FWHM) de 1.16eV. Las muestras fueron desecadas en alto vacío durante varias horas antes de pasar a la cámara de análisis, donde el vacío fue siempre menor de $4.0 \times 10^{-7} \mathrm{~Pa}$. La composición química de la superficie de las membranas se determinó usando las relaciones de áreas de los picos principales de cada elemento, pesadas con los factores de sensibilidad suministrados con el software del equipo (6), y tras realizar la sustracción integrada del fondo (7).

\section{RESULTADOS Y DISCUSION}

\subsection{Determinación de los parámetros electroquímicos}

La Fig. 1 muestra la variación con la concentración de electrolito $(\mathrm{NaCl})$ de la resistencia de la capa de proteína. La disminución de la $\mathrm{R}_{\mathrm{BSA}}$ al aumentar $\mathrm{C}_{\mathrm{NaCl}}$ es debida a la dependencia con la concentración de la disolución contenida en la capa de proteína. La relación $\mathrm{R}_{\mathrm{AnL}} / \mathrm{R}_{\mathrm{AnF}}$ indica una reducción aproximada del $60 \%$ en la porosidad de la membrana, lo que ha sido confirmado con resultados obtenidos mediante microscopía de fuerza atómica (AFM) (8).

En la Fig.2 se muestra una comparación de la difusión salina a través de una membrana límpia y otra colmatada, tras estar en contacto durante tres días con la disolución conteniendo BSA. Debido a la adsorción de proteína sobre la membrana, la pendiente de la curva concentración-tiempo se ha reducido, aproximadamente, a la mitad. Puesto que la permeabilidad de una membrana porosa, $\mathrm{P}_{\mathrm{S}}=\mathrm{J}_{\mathrm{S}} / \Delta \mathrm{C}$, está relacionada con el coeficiente de difusión de la sal, $\mathrm{D}_{\mathrm{S}^{\prime}}$ el espesor de la membrana, $\Delta \mathrm{x}_{\mathrm{m}^{\prime}}$ y su porosidad, $S_{p^{\prime}}$ mediante la expresión (9): $P_{s}=\left(D_{s} S_{p} / \Delta x_{m}\right)$, la reducción a la mitad de la permeabilidad salina supone una reducción del $50 \%$ de la porosidad de la membrana (se desprecia el espesor de la capa de proteina), lo que está de acuerdo con los resultados indicados anteriormente. 


\subsection{Análisis de los espectros de XPS}

La adsorción de la proteína sobre la membrana de alúmina se puede determinar fácilmente mediante el incremento de las concentraciones atómicas de $\mathrm{C}$ y $\mathrm{N}$ (elementos característicos de la proteína, pero no de la membrana) y el descenso de las concentraciones de $\mathrm{Al}$ y $\mathrm{O}$ (elementos característicos de la membrana). En la Fig. 3 se ha representado el espectro de XPS correspondiente al nivel electrónico $\mathrm{Al} 2 \mathrm{p}$ para distintos tiempos de interacción membrana-proteina; la drástica disminución del área con el tiempo es un índice del recubrimiento de la superficie de la membrana de alúmina por la proteína. En la Tabla I se indican las concentraciones atómicas de los elementos existentes en la superficie de las membranas. Puesto que el $\mathrm{C}$ y $\mathrm{N}$ no son elementos constitutivos de las membranas de alúmina, deben de proceder de contaminación o impurezas superficiales de la muestra utilizada (al igual que un $2 \%$ de $\mathrm{P}$ no indicado); la presencia de estas impurezas, que previsiblemente también contienen oxígeno, hace que la relación $\mathrm{Al} / \mathrm{O}$ obtenida sea menor de la teórica $\left([\mathrm{Al} / \mathrm{O}]_{\text {teorica }}=0.667\right)$. La variación temporal de las concentraciones indicadas Tabla I, muestra como la adsorción de proteína sobre la superficie de la membrana de alúmina no se produce de forma lineal, sino que es mucho más rápida en las primeras horas. Consecuentemente con esta variación de las concentraciones, las relaciones $\mathrm{Al} / \mathrm{O}$ y Al/ $\mathrm{N}$ disminuyen al aumentar el tiempo que permanece la membrana en contacto con la BSA, mostrando también el recubrimiento de la alúmina por la proteína. Supuesto que la profundidad de análisis mediante XPS es menor que el tamaño de la proteina (69.000 Dalton), los resultados anteriores indican que la proteina no forma una capa uniforme capaz de cubrir la membrana, sino que deja huecos que permiten detectar mediante XPS una cierta cantidad de aluminio.

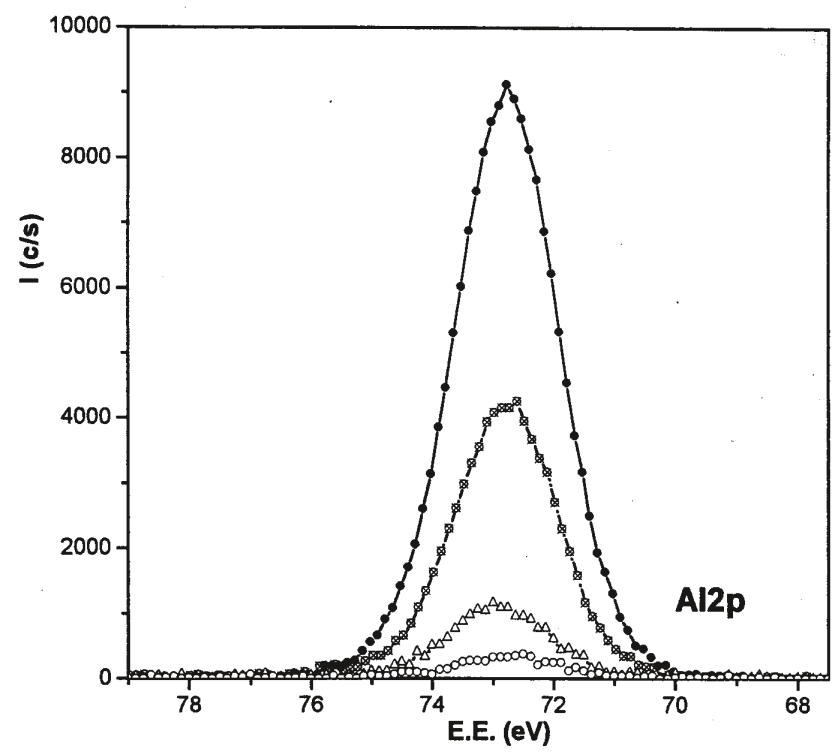

Figura 3: Espectros de XPS correspondiente al nivel electrónico Al 2p para distintos tiempos de interacción membrana-proteína. (o) $\mathrm{t}=0$, $(\mathrm{O})$ $\mathrm{t}=24 \mathrm{~h}$; ( ) $\mathrm{t}=48 \mathrm{~h}$ and (o) $\mathrm{t}=72 \mathrm{~h}$.
TABLA I. CONCENTRACIONES ATÓMICAS DE LOS ELEMENTOS CARACTERÍSTICOS DE LAS MEMBRANAS ANL Y ANF EN FUNCIÓN DEL TIEMPO DE INTERACCIÓN MEMBRANA-PROTEINA.

\begin{tabular}{ccccccc}
\hline Membrana & $\% \mathrm{C}$ & $\% \mathbf{O}$ & $\% \mathbf{A l}$ & $\% \mathbf{N}$ & Al/O & Al/N \\
\hline AnL & $19.4^{*}$ & 53.0 & 26.4 & $1.2^{*}$ & 0.501 & 22.64 \\
AnF-24 h & 44.7 & 30.8 & 13.4 & 10.6 & 0.450 & 1.303 \\
AnF-48 h & 61.6 & 25.8 & 3.4 & 9.3 & 0.130 & 0.362 \\
AnF-72 h & 64.2 & 24.7 & 1.2 & 9.9 & 0.048 & 0.120 \\
\hline
\end{tabular}

"Estos elementos se atribuyen a impurezas superficiales de ta membrana de alúmina.

\section{CONCLUSIONES}

La colmatación de una membrana comercial de alúmina para microfiltración, debida a la adsorción/deposición de proteína sobre su superficie, se ha estudiado mediante la variación de algunos parámetros electrocinéticos y el análisis químico de la superficie de membranas límpias y colmatadas.

- La reducción de la porosidad de la membrana colmatada se ha puesto de manifiesto mediante: i) el aumento de la resistencia eléctrica; ii) la disminución de la difusión salina.

- El análisis químico de la superficie muestra que no existe un recubrimiento uniforme de la superficie de la membrana por la proteína. Un estudio de la colmatación con el tiempo de interacción membrana-proteina muestra una cierta saturación en la cantidad de proteína adsorbida a partir de 48 horas.

\section{AGRADECIMIENTOS}

Agradecemos a la Comisión Interministerial de Ciencia y Tecnología (CICYT) su ayuda económica (Proyecto MAT0970-C03-01).

\section{BIBLIOGRAFIA}

1. M.Mulder, Basic Principles of Membrane Technology, Kluwer Academic Publishers, 1991.

2. M.Nyström, "Fouling of unmodified and modified polysulfone ultrafiltration membranes by ovalbumin", J.Membrane Sci., 44, 183-196, 183. (1989).

3. J.Benavente y G.Jonsson, "Effect of adsorption of charged macromolecules on streaming and membrane potential values measured with a microporous polysulfone membrane", Sep.Sci.\&Technol., 32, 1699-1710 (1997).

4. A.Cabeza, M.Martinez, J.Benavente y S.Bruque, "Current rectification by $\mathrm{H}_{3} \mathrm{OUO}_{2} \mathrm{PO}_{4} \cdot 3 \mathrm{H}_{2} \mathrm{O}$ (HUP) thin films in electrolyte media", Solid State Ionics, 51, 127-131 (1992).

5. T.Takanishi, T.Akiyama, T.Seike, M.Satoh y J.Komoyama, “Electrical conductivity of cation poly (vinyl alcohol) membranes having quaternary ammonium groups", J.Membrane Sci., 64, 247-254 (1991).

6. J.F.Moulder, W.F.Stickle, P.E.Sobol, K.D.Bomben, Handbook of X-ray photoelectron spectroscopy (Perkin-Elmer Corp.), 1992.

7. D.A.Shirley, "High resolution X-ray photoemission spectrum of the valence bands of gold", Physical Review B, 5, 4709-4714 (1972).

8. J.Benavente, L.Palacios, P.Prádanos, J.I.Calvo, y A.Hernández; Ceramics: Getting into the 2000's, part D, TECHNA (editor: P.Vicenzini), 503-508 (1999).

9. J.Benavente, M.I.Vazquez-Gonzalez y J. de Abajo, "Effect of UV light on different structural and transport parameters of cellophane membranes", Sep. Sci. Technol., 31, 189-199 (1996). 